\title{
Momentum and default risk. Some results using the jump component
}

April 2015

\begin{abstract}
:
In this paper we separate the total stock return into its continuous and jump component to test whether stock return predictability should be attributed to omitted risk factors or behavioral finance theories. We extend results from the US market to the Spanish stock market, which, despite being a developed market, presents several differences in terms of stock characteristics, financial system, investor typology and cultural dimensions. The results show that the jump component has significant explanatory power for the premium of three characteristics (size, book-to-market and illiquidity), which is at odds with risk-based explanations. Using the same testing strategy, we try to shed some light on an important controversy concerning the relationship between default risk and momentum. The results suggest that default risk is not the source of momentum returns.
\end{abstract}

Keywords: Jumps, Momentum, Default Risk, behavioral finance

JEL Code: G12, G14, C10

\section{ACKNOWLEDGEMENTS}

This paper has received financial support from the Spanish Ministry of Economy and Competitiveness (ECO2012-35946-C02-01). Ana González-Urteaga particularly acknowledges the financial support of the Spanish Ministry of Economy and Competitiveness (ECO2012-34268).

Ana González-Urteaga. Dpto. Gestion de Empresas. Universidad Publica de Navarra. Campus de Arrosadia s/n 31.006 Pamplona (SPAIN). E-mail: ana.gonzalezu@unavarra.es

Luis Muga. Dpto. Gestion de Empresas. Universidad Publica de Navarra. Campus de Arrosadia s/n 31.006 Pamplona (SPAIN). E-mail: luis.muga@unavarra.es

Rafael Santamaria. (Corresponding author). Dpto. Gestion de Empresas. Universidad Publica de Navarra. Campus de Arrosadia s/n 31.006 Pamplona (SPAIN). 34948169389 E-mail: rafael@unavarra.es 


\section{Momentum and default risk. Some results using the jump component}

\section{Introduction}

Stock return predictability has been related to various stock characteristics, such as size, Book-to-Market (btm), liquidity, and momentum, among others. These relationships have been explained from risk-based and behavioral perspectives. On the one hand, Fama and French $(1992,1996)$ claim that size and value premiums are risk factors omitted from models such as the CAPM, a view supported by other authors, such as Jagannathan and Wang (1996) and Zhang (2006). Meanwhile, from the behavioral finance point of view, characteristics such as small size, extreme btm or low liquidity can be interpreted as proxies for stocks with high information uncertainty (Jiang et al., 2005), firms that are hard to value or to arbitrage (Baker and Wurgler, 2006), or stocks attracting limited attention (Abody et al., 2010).

Jiang and Yao (2013) offer a new way to distinguish between these two competing explanations by separating total stock return into its continuous and jump components. These authors assert that, if investors form unbiased rational expectations, the unexpected component of stock return should not be correlated with ex ante firm characteristics. However, if they form biased expectations and this bias is related to stock characteristics, this component will be associated with lagged characteristics, due to price reversion to equilibrium. In this vein, it is widely accepted in financial literature that large discontinuous price changes (price jumps), due to news or liquidity shocks, are rare events that can proxy for significant information shocks. Hence "if the predictable component of stock returns is realized in the form of jumps that occur on average only twice a year, such predictability is likely driven by investors' response to new information arrival and unlikely to be the effect of risk Premium" (Jiang and Yao, 2013, pp 1519-1521). The results obtained by these authors for the US market indicate that size and illiquidity effects, along with a significant part of the value premium, are realized in the form of jumps, thus bringing into question risk premium-based explanations for cross-sectional stock return predictability based on these variables. Furthermore, neither jump risk nor the "martingale restriction"1 on jumps can explain their results.

\footnotetext{
1 Since a martingale is a zero-drift stochastic process, the literature often imposes the martingale restriction on jumps. This means that, in a rational continuous- time asset-pricing model, the jump component in the
} 
In this context, it is worth analyzing whether these results are stock market-dependent or can be generalized to other markets. For this purpose and following the same methodology as Jiang and Yao (2013), we perform the analysis for the Spanish stock market because, despite being a developed financial market, it presents major differences in key variables that drive stock prices. Firstly, in particular relation to stock characteristics, spreads are lower in the Spanish market than in the US market, and this probably causes a decrease in their premiums. Secondly, average firm size is bigger in the Spanish market ${ }^{2}$. This is an important issue, since stock characteristics have the potential to change or accentuate investors' behavioral biases. Some behavioral finance models (see, among others, Daniel et al., 1998 and 2001 or Hirshleifer, 2001) have shown that investors' behavioral biases are stronger among relatively hard-to-value stocks operating in informationally-sparse environments. The type of financial system can also affect stock price behavior due to the potential effect of the degree of shareholder dispersion and separation between ownership and control. In these terms, the US market is Anglo-Saxon, that is, it is characterized by shareholder dispersion and a wider separation between ownership and control. Spain, on the other hand, has a continental system, characterized by a more highly concentrated ownership structure. In addition to that and concerning the investor typology in Spain the majority of institutional investors are banks, which have a more stable relationship with firms, whereas, in the AngloSaxon system, they are mainly mutual funds or pension plans (MF\&PP) and, although institutional investors are sophisticated traders, and their presence contributes to efficient asset pricing due to their superior capacity to acquire and process information (see, among others, Bartov et al., 2000, Jiambalvo et al., 2002 or Collins et al., 2003) they are not a homogeneous group. In fact, MF\&PP can be defined as "transient" institutions because they may overweight short-term earnings potential and underweight long-term earnings potential due to their incentives and may pressure managers into a short-term focus (see Bushee, 2001), whereas banks generally have a long-term, stable relationship with firms. Finally, the US and Spain also display contrasting cultural profiles based on the dimensions coined by Hofstede $(2001)^{3}$, and cultural dimensions have been found to play a major role in explaining the impact of investor behavior on market prices (see Schmeling, 2009 or Corredor et al., 2013).

drift term captures expected jumps and, consequently, the difference between realized jumps and the jump component forms a martingale with zero expectation (see Merton, 1976).

2 According to Datastream data for 2011 the New York stock exchange lists both very small and very large firms. However, the average size of the firms listed in the Spanish stock market is approximately three times that of those listed in the NYSE.

3 The US/Spanish scores on the five dimensions are Power Distance (40/57), Individualism (91/51), Masculinity (62/42), Uncertainty avoidance (46/86) and Long-term orientation (29/19). 
In addition, using the same testing strategy as Jiang and Yao (2013), we aim to shed light on another key controversy not studied in the cited paper. In particular, we study the origin of momentum returns and its relation with default risk, which is the most innovative aspect of this paper. While Avramov et al. (2007) and Agarwal and Taffler (2008), using different default risk proxies, conclude that default risk is the key variable to explain the momentum effect, Abinzano et al. (2014) find no empirical support for this claim. They identify default risk as simply one more characteristic of the loser portfolio, which lacks sufficient explanatory power, on its own, to account for total return. Following the logic applied by Jiang and Yao (2013), if default risk is a key factor in the momentum effect, both these characteristics will necessarily show significant premiums on the same (jump or continuous) return component. Otherwise, it can hardly be argued that momentum and default risk are directly related or that one is the direct cause of the other.

Thus, this paper makes two main contributions to the literature. First, we test whether Jiang and Yao's (2013) findings for US are stock-market-dependent, analysing the Spanish market, which presents significant differences in aspects such as the firm characteristics, financial system, investor typology and cultural dimensions. Our results show that these differences have major repercussions on the frequency and intensity of jumps, both of which are lower in the Spanish stock market. However, the main conclusions hold and thus, our findings strengthen the robustness and generalizability of those of Jiang and Yao (2013). Second, and more importantly, we use the same testing strategy in order to see whether default risk is a potential explanation for the momentum effect. Our results shed some light on this controversy by confirming the conclusions reached by Abinzano et al. (2014) and enabling us to conclude that default risk is not the source of momentum returns, given that the predictable component of returns manifests itself differently in each case: as jumps in the case of default risk and as continuous returns in that of momentum.

The remainder of the paper is organized as follows: Section 2 describes the statistical procedures used to identify jumps and break down stock returns into their continuous and jump components. Section 3 presents the database and preliminary data analysis. Section 4 summarizes the empirical findings from the analysis of stock characteristics and return predictability, and reports on various robustness checks based on a reestimation controlling for liquidity shocks, jump risk and the "martingale restriction" on jumps. Section 5 analyzes the continuous and jump components of total stock returns to 
explore the relationship between the momentum effect and default risk. Finally, section 6 presents the paper's main conclusions.

\section{Jump Measure}

From all the statistical jump detection options ${ }^{4}$ proposed in literature, we select the one developed by Jiang and Oomen (2008). Our reasons for this choice are basically threefold. Firstly, it is a powerful method for identifying large, infrequent jumps in returns and provides reliable estimates of jump returns. Secondly, it is more sensitive to jumps than other tests because it uses both second and higher-order moments. Jiang and Oomen (2008) provide simulations which show their statistic to perform comparatively well. Thirdly, the approach is model free, as it does not impose functional specifications on the drift, diffusion or jump dynamics. Moreover, its implementation requires only asset prices. Hence, its implementation on a large cross-section of stocks over a long time period is straightforward.

This test is inspired by the variance swap, a contract whose payoff depends on the realized variance. Intuitively, it reflects the cumulative gain of a variance swap replication strategy, which is minimal (substantial) in the absence (presence) of jumps. Specifically, when there is no jump, the difference between simple returns and log returns equals one half of the instantaneous return variance (see Jiang and Oomen, 2008 for a detailed, technical explanation). Based on this result, the variance swap replication strategy is perfect. However, when jumps occur, the difference is also a function of realized jumps and the aforementioned replication strategy fails. The method is based on precisely this insight.

Let $S_{t}$ be the price of a stock at day $t$, where $R_{t}=\left(S_{t}-S_{t-1}\right) / S_{t-1}$ is the simple return and $r_{t}=\ln \left(S_{t} / S_{t-1}\right)$ denotes the continuously compounded or log return. The variance swap measure is computed as

$$
S V_{T}=2 \sum_{t=1}^{T}\left(R_{t}-r_{t}\right)
$$

where $T$ denotes the sample size, whereas the realized variance is defined as

\footnotetext{
${ }^{4}$ For instance, Barndorff-Nielsen and Shephard (2006) develop a test based on bipower variation. A modified version of this test is used by Huang and Tauchen (2005) and Andersen et al. (2007). Lee and Mykland (2008) also use bipower variation but in a different way, developing a nonparametric statistical test of jumps. Aït-Sahalia and Jacod (2009) and Tauchen and Zhou (2011) propose nonparametric tests for presence of jump.
} 


$$
R V_{T}=\sum_{t=1}^{T} r_{t}^{2}
$$

which is known to converge to the total variation of the process. Thus, by construction, the difference between the $S V_{T}$ and $R V_{T}$ values can be used to detect the presence of jumps. As Jiang and Oomen (2008) prove, since the price process is modeled as discrete time observations, the difference between the two values can be used to test statistically whether or not jumps have occurred. More precisely, when jumps are absent, their ratio will be indistinguishable from one, but when they are present it will reflect the replication error of the variance swap. Under the null hypothesis of no jumps over the period $[0, T]$, Jiang and Oomen (2008) proposed the following ratio test:

$$
J T=T \frac{V_{(0, T)}}{\sqrt{\Omega_{S V}}}\left(1-\frac{R V_{T}}{S V_{T}}\right) \stackrel{d}{\rightarrow} N(0,1)
$$

where $V_{(0, T)}=\int_{0}^{T} V_{t} d t$ where $V_{t}$ is the diffusive variance when there is no random jump, and $\Omega_{S V}=\frac{1}{9} \mu_{6} \int_{0}^{T} V_{t}^{3} d t$ with $\mu_{p}=2^{p / 2} \Gamma((p+1) / 2) / \sqrt{\pi}$ for $p>0$ where $\Gamma(\cdot)$ denotes the gamma function. Consistent estimates of $V_{(0, T)}$ and $\Omega_{S V}$ are given by bi-power variation

$$
\widehat{V}_{(0, T)}=B P V_{T}=\frac{1}{\mu_{1}^{2}} \frac{T}{T-1} \sum_{t=1}^{T-1}\left|r_{t} r_{t+1}\right|
$$

and multi-power variation

$$
\widehat{\Omega}_{S V}^{(p)}=\frac{\mu_{6}}{9} \frac{T^{3} \mu_{6 / p}^{-p}}{T-p+1} \sum_{t=0}^{T-p} \prod_{k=1}^{p}\left|r_{t+k}\right|^{6 / p}
$$

respectively, where $p$ is equal to $\operatorname{six}^{5}$.

After identifying jump days ${ }^{6}$, jump and continuous return components are disentangled from total stock returns each year. Let $T R$ be the total annual stock return and $\left\{r_{k}^{J}\right\}_{k=1}^{K}$ the log of identified jump returns during the year. The jump (JR) and continuous (CR) returns are computed as

$$
J R=\exp \left(\sum_{k=1}^{K} r_{k}^{J}\right)-1 ; \quad C R=T R-J R
$$

Following Jiang and Yao (2013), we also construct a so-called liquidity-adjusted jump return, $L J R$, where each jump day's return is now calculated by aggregating returns from

\footnotetext{
${ }^{5} p$ must be 4 or 6 for the robust estimation of $\Omega_{S V}$ (Jiang and Oomen, 2008). We perform the analysis for both values and the results are consistent. Therefore, we show only those corresponding to $p$ equal to six (the rest are available upon request).

6 The sequential approach used by Jiang and Yao (2013) is adopted to identify days with stock price jumps. See Appendix A for further details about this procedure.
} 
$k$ to $k+47$. This measure should mitigate the effect of liquidity shocks, taking into account that this kind of jumps will revert over subsequent trading days. Likewise, the liquidityadjusted continuous return is computed as

$$
L C R=T R-L J R
$$

Finally, the total jump risk of stock returns is constructed annually by

$$
J R i s k=R V-B P V
$$

a measure that captures jump return variance. Barndorff-Nielsen and Shephard (2004) develop this measure after decomposing the return variance into the continuous component of log-prices and the impact of jumps. This decomposition is crucial when measuring the risk associated with an asset, since it allows us to distinguish between diffusion risk and jump risk. The latter is the one used in equation (8).

\section{Data and Preliminary Analysis}

Given that behavioral finance theories have emphasized the role of variables such as type of financial system, investor typology, stock characteristics, and cultural dimensions on stock prices, the key question is whether the results of Jiang and Yao (2013) for US data are market-dependent or can be generalized to other markets. To explore this issue, we have chosen the Spanish stock market because, although it is a developed financial market, it displays major differences in all of the above variables.

The study data were obtained from the Thomson Financial ${ }^{8}$ database, where from all the stocks listed on the Spanish continuous market (SIBE), we were able to collect daily return data for 208 stocks listed on the Spanish stock exchange between January 1990 and July 2011. Using a significance level of $1 \%{ }^{9}$, we performed the above econometric jump test on daily stock returns over each calendar quarter ${ }^{10}$. This requires a minimum

\footnotetext{
${ }^{7}$ Although period $\mathrm{k}$ to $\mathrm{k}+4$ is used for consistency with Jiang and Yao (2013), the conclusions also hold for $\mathrm{k}$ to $\mathrm{k}+3$ and $\mathrm{k}$ to $\mathrm{k}+5$. These results are not shown but are available upon request.

8 Various filters suggested by Ince and Porter (2006) were applied to avoid bias from the naive use of the Thomson Datastream database. In particular, we have removed padded zero-return records at the end of delisted firms, all nonlocal firms, all listings other than those on the primary exchange and all listings with Type not equal to Equity. We include only those firms that checked YES in the "Primary quote" field.

9 Results (available upon request) are robust if we focus mainly on the large jumps by changing the critical value of the jump test to 3 or 4 (e.g. Andersen et al., 2007 and Huang and Tauchen, 2005).

${ }^{10}$ Although the literature seems to focus on very high frequency data in calculating statistical measures of jumps, following Jiang and Yao (2013) we focus on daily data for several reasons. Availability of intra-day data is limited. Besides, daily frequency helps mitigate market microstructure noise and avoids the need for the corresponding modified Jiang and Oomen (2008)'s test for high frequency data. In fact, Christensen et al.
} 
of 44 daily return observations during the quarter, thus, 176 observations to calculate the total jump risk given by (8). If the test is unfeasible, a zero jump return is recorded. To avoid the misinterpretation of no trading activity, any observations for which the previous three days' return data are not available are not classed as jumps.

A brief summary statistics of jumps is shown in Table 1. The jump frequency for each stock is computed in annual terms (from July of year $t$ to June of year $t+1$ ) as the ratio of the total number of jumps to the number of years the stock is in the sample. Jump size is the average in absolute value of all realized jumps. We also compute the jump size for positive and negative jumps. The mean and median jump frequencies are 1.11 and 1.00, which is clearly lower than those obtained by Jiang and Yao (2013) for the US market (2.02 and 2.00, respectively for the most recent sample period 1962-2009). The mean and the median of the absolute jump sizes are $9.87 \%$ and $8.82 \%$, respectively. Once again, these figures are lower than those obtained for the US market (14.24\% and 11.84\%). The jumps estimated at $5^{\text {th }}$ and $95^{\text {th }}$ percentiles in the Spanish stock market $(4.55 \%$ and $19.32 \%$ ) are both clearly lower than in the US market (5.49\% and $30.76 \%$ ).

The statistics for positive and negative jumps reveal that the positive jumps are notably more frequent (0.84) than the negative ones (0.26), which is in line with the findings reported by Jiang and Yao (2013) and consistent with the average positive skewness in daily stock returns documented in the literature (see Campbell et al., 1997). However, it is important to note that the average size of negative jumps $(-11.21 \%)$ is greater than that of positive ones $(9.52 \%)^{11}$.

These results show that the differences between the two markets have a not negligible impact on the jump distribution and intensity. In particular, the lower frequency and smaller size of jumps in the Spanish stock market could be due to various reasons. These include differences in firm size, because the smallest firms in the Spanish stock market are not listed; differences in ownership concentration, because in Spain ownership is clearly more highly concentrated; and differences in investor typology, because, in contrast to the US market, where they are MF\&PP, the main institutional investors in

(2011) show that many intra-day jumps often quickly revert, since they are caused by market microstructure noise or liquidity. Daily data is also useful for picking out infrequent and large jumps over a holding period of one year. Daily frequency seems indeed acceptable since the majority of investors do not rebalance continually, or even daily. Finally, the jump test is performed quarterly to take into account time-varying volatility of stock returns (see Jiang and Yao, 2013, for more details).

${ }^{11}$ As a robustness check, we define as a jump any return which is smaller (bigger) than the mean minus (plus) $k$ standard deviations and this pattern holds: positive jumps are more frequent but smaller than the negative ones. This result holds for $k=1.5,2,2.5$ and 3. Furthermore, both patterns become clearer with increases in the value of $k$. 
Spain are banks, which have more a stable relationship with firms. The major differences observed in the jump distribution further fuel our aforementioned interest in testing whether they might significantly influence our conclusions as to whether or not jumps, as a proxy for the unexpected return component, are correlated with ex-ante firm characteristics and whether this might alter the explanatory power of risk-based and behavioral explanations of stock return predictability.

The size and btm variables are based on monthly data drawn from the Thomson Financial database. Size is computed as the logarithm of market capitalization and btm is the ratio between the book value and the market value. Liquidity is proxied by the illiquidity measure developed by Amihud (2002), which is the average ratio of the absolute daily return to the (monetary unit) trading volume on that day:

$I L_{i, t}=\frac{1}{D_{i, t}} \sum_{d=1}^{D_{t}} \frac{\left|R_{i, t}\right|}{V_{i, t}}$

where $D_{i, t}$ is the number of days for which data are available for stock $i$ in month $t$, and $R_{i, t}$ and $V_{i, t}$ denote the daily return and daily trading volume (in euros), respectively, at day $t$.

Table 2 reports the descriptive statistics of jumps in portfolios sorted by size, btm, illiquidity ratio and momentum ${ }^{12}$. Most of the jumps found in the size-sorted portfolios are in the smallest firm quintile and the data show a decreasing monotonic pattern overall, except in the second quintile and average jump size is significantly greater for small firms than for big ones.

In the btm-sorted portfolios, the number of jumps follows a u-shaped curve and average jump size an inverted u-shaped curve. However, there are no differences between the extreme quintiles either in the number or the average size of jumps. The only significant difference is the larger number of negative jumps in the low btm ratio quintile.

With the exception of quintile 4, the illiquidity ratio quintiles show an increasing monotonic pattern both in the number and average size of jumps, with the highest

\footnotetext{
${ }^{12}$ In their seminal paper, Jegadeesh and Titman (1993) use combinations of formation periods $(\mathrm{J}=3,6,9$ and 12 months) and holding periods ( $\mathrm{K}=3,6,9$ and 12 months) making a possible total of 16 different momentum strategies. Later literature takes as reference the $\mathrm{J}=6, \mathrm{~K}=6$ strategy. For examples, see Rouwenhorst (1998) or Griffin et al. (2003). We too have computed this 6X6 momentum strategy. Thus, in the case of the momentum characteristic, stocks are sorted by the past 6 months' cumulative return.
} 
values appearing in the more illiquid stocks. The difference between the extreme quintiles is significant at conventional levels except in the case of negative jumps.

While there is no clear pattern in the number of jumps in the momentum portfolio, the average size follows a u-shaped curve. However, neither in the number nor in the average size of jumps is there a significant difference between the two extreme quintiles, winners minus losers. These results suggest that the variables most strongly related to jumps are size and liquidity. They also clearly illustrate that, irrespective of the portfolio-sorting variable, positive jumps are more frequent but smaller in average absolute value than negative ones.

Finally, following Vassalou and Xing (2004) and Abinzano et al. (2014), we use the Black-Scholes-Merton (BSM) measure as a proxy for default risk. We use this measure instead of the credit rating because there is no available credit rating for several stocks in the Spanish stock market and a firm's credit worthiness can vary substantially before its credit rating is readjusted. We use a market-based measure because accountingbased measures have certain disadvantages, particularly stemming from the failure to consider asset volatility, as a result of which firms with the same ratios are attributed with exactly the same likelihood of going bankrupt. This measure of default risk is given by the following expression:

$$
P_{d e f, t}=N\left(-\frac{\ln \frac{V_{A, t}}{D_{t}}+\left(\mu_{t}-\frac{\sigma_{A, t}^{2}}{2}\right)(T-t)}{\sigma_{A, t} \sqrt{T-t}}\right)
$$

where $V_{A, t}$ is the value of firm assets at time $t, \mu_{t}$ is the expected immediate rate of return on $V_{A, t}, \sigma_{A, t}$ is asset return volatility, $D_{t}$ is the debt face value, $T$ is the maturity period and $N(\cdot)$ is the cumulative probability of the normal distribution. To find the values of $V_{A, t}$ and $\sigma_{A, t}$, we use an iterative process starting from the market price of the firm's shares (See Vassalou and Xing, 2004 for further details) ${ }^{13}$. We use debt data for

\footnotetext{
${ }^{13}$ However, the value of the firm's assets is not directly observable and nor, therefore, are the volatility or the average rate of return. The one observable variable is the market value of equity, which can be used to estimate the volatility of its return. By solving the equation $\sigma_{E, t}=V_{A, t} / V_{E, t} N\left(d_{1}\right) \sigma_{A, t}$ iteratively, starting with the market value of equity, it is possible to calculate the value of the firm's assets, volatility and mean return. We use a tolerance level of 0.001 and adopt the usual forecasting horizon of 1 year $(T=1)$.
} 
the last three quarters of the current year and the first quarter of the past year and calculate the book value of debt as the sum of short-term debt and $50 \%$ of long-term debt.

Shares in banks and finance and insurance companies were excluded from the construction of the default measure, because the difference in their capital structure could skew the resulting default risk estimates. This reduced the sample to 124 shares for this part of the analysis, causing a degree of sample bias. This has little consequence, in the case in hand, however, because, as shown by Muga and Santamaría (2007), the estimates of momentum returns from finance sector stocks in this market are not statistically significant.

\section{Empirical results}

\subsection{Cross-sectional return predictability}

In this section, we analyze return predictability based on the four characteristics named above (size, btm, momentum, and illiquidity ratio). In June of year $t$ stocks are sorted by a specific characteristic and the return for the holding period from July of year $t$ to June of year $t+1$ is then computed. Then returns are aggregated in equal-weighted quintile portfolios. In the case of the momentum portfolios, both the formation period (from January to June of year $t$ ) and the holding period (from July to December of year $t$ ) are 6 months long.

Table 3 shows the return of the four characteristic-based portfolio quintiles and the return spread between their top and bottom quintiles. All of these spreads show the expected sign but, in contrast to the results obtained by Jiang and Yao (2013) for the US stock market, this spread is significant at conventional levels only for the btm and momentum portfolios. The lack of significance of the premium for the size and illiquidity ratio is not exceptional in the market analyzed, due to the high average firm size of SIBE listed companies, as small and medium firms are not listed in this market.

However, the lack of significant premiums associated with size and illiquidity does not rule out the possibility of the jump component having a significant premium offset by the continuous return component (or vice versa), thus resulting in the lack of significance found for the total returns. We clarify this issue with the data displayed in Table 4, which shows the premiums obtained for the 4 characteristics of interest after separating the continuous component (CR) from the jump component (JR) in total returns. This 
enables us to find significant jump premiums in the size-sorted portfolio returns $(6.67 \%$ per year) and the illiquidity-sorted portfolio returns (9.92\% per year). The fact that these premiums are partially offset by the CR in both these portfolios is the reason for the lack of significance of the premiums observed in Table 3.

The behaviour of the btm premium differs from that of the two variables discussed above, in that both the jump return (3.86\% per year) and the continuous return $(8.33 \%$ per year) are statistically significant.

Thus, the results enable us to sustain the claim that the return predictability of stocks with the characteristics of interest in the Spanish stock market cannot be attributed solely to risk-based explanations, given that, if investors form unbiased rational expectations, the unexpected component of stock return should not be correlated with ex ante firm characteristics. These results are consistent with those obtained by Jiang and Yao (2013) for the US stock market.

Finally, the momentum premium behaves differently from the rest of the study variables, in that the continuous return component shows a six-month premium of $10.48 \%$, while the jump return component is negative with a value of $-1.87 \%$. This separation between JR and CR components is very similar to that in the US stock market.

These results show that, although the literature has repeatedly associated the momentum effect with liquidity, size and btm, momentum returns arise from the CR component, while liquidity and size returns come mainly from the JR component. Thus, momentum definitely behaves differently from size and liquidity, since the former is related to the effect of the risk premium (the continuous component) and the latter to the unexpected component of stock returns and is therefore unlikely to be related to the risk premium. The btm is the only characteristic to show a significant premium in the CR component (as well as in the JR component) which would suggest that momentum and btm could be related, as claimed by Daniel and Titman (1997).

A final point worth noting is that these results show that jump frequency and average jump size are both lower in the Spanish market, probably because of characteristic differences with other markets (specifically, larger average firm size, higher ownership concentration, and a stronger presence of institutional investors from the banking sector whose relationships with the business world are more stable), the main conclusions 
largely coincide with those of Jiang and Yao (2013) for the US stock market, thereby strengthening the robustness and generalizability of their findings.

\subsection{Robustness checks}

This section describes the various robustness checks performed in this study. First, returns are calculated for firms with jumps and firms without jumps. For every year $t$, the "firms with jumps" sample includes all firms presenting at least one jump over the formation period July $t-1$ to June $t$. The returns are then calculated for the holding period from July $t$ to June $t+1^{14}$. The remaining firms make up the "firms without jumps" sample. The results are in line with those obtained in the previous section (see Table 5). In particular, the yearly size premium of $-12.46 \%$ and the yearly liquidity premium of $13.25 \%$ are significant only in the "firms with jumps" sample, while the sixmonth momentum premium of $7.61 \%$ is significant only in the "firms without jumps" sample. In both samples, the yearly book-to-market premium is significantly positive: $13.76 \%$ in the "with jumps" sample and $10.61 \%$ in the "without jumps" sample.

Next, we adjust for liquidity shocks in asset prices by constructing the "liquidityadjusted" jump return LJR and the corresponding "liquidity-adjusted" continuous return LCR described in Section 2. LJR is the originally identified jump return compounded with the stock returns over the subsequent four trading days to overcome potential stock price reversion, and LCR is the difference between total return and LJR. This enables us to mitigate the effect of temporary liquidity shocks on our empirical results. The results (see Table 6) remain the same as those reported in Table 4 for the size, liquidity, and momentum premiums. Jumps fully account for the size and liquidity effects, but they do not drive momentum. The difference emerges in the btm variable. After adjusting for liquidity, the jump premium ceases to be significant, and only the continuous component has a significantly positive premium. This result suggests that liquidity jumps do, in fact, account for a significant part of the value premium.

We then repeat the exercise while controlling for jump risk using a double-sorting procedure. Stocks are first sorted into tertiles ${ }^{15}$ based on the jump risk measure JRisk. Then, within each tertile, stocks are further sorted into tertiles based on one of the four characteristics (size, btm, momentum, and illiquidity ratio). The new tertile portfolios are obtained by combining all the stocks with the same characteristic tertile rank across

\footnotetext{
${ }^{14}$ As in the previous section, six-month formation and holding periods are used for the momentum portfolios.

${ }^{15}$ Because the total number of stocks in the Spanish stock market is not large enough to use quintiles, we use tertiles instead to allow some degree of diversification in the final portfolios.
} 
three jump risk tertiles, and computing equal-weighted portfolios. This procedure allows each tertile portfolio to capture a different stock characteristic but the same jump risk. The results obtained are very similar to those obtained without controlling for jump risk (see Table 7). In this case, although the total premiums maintain the expected sign, they lack significance for all the variables. Size shows a premium in the continuous component. This premium is also shown for the momentum effect. The results for the JR follow the same pattern as before with significant premiums for size (-7.00\% per year), BTM (3.43\% per year) and liquidity (8.69\% per year). The results after controlling for liquidity are basically the same. In summary, after controlling for jump risk, the predictive pattern of size and liquidity premiums realized by jumps remains unaltered.

Finally, we also take into account the implications of the "martingale restriction" on jumps. In continuous-time models with random jumps the literature often imposes a restrictive form for the instantaneous drift, in order to form a martingale with zero conditional expectation (see, e.g., Merton, 1976; Andersen et al., 2002, and Pan, 2002). This martingale restriction on jumps under the rational asset-pricing models implies that, even after controlling for jumps, the predictability of firm characteristics is expected to be maintained. To test this implication, we use another double-sorting procedure to control for jumps. First, in June of year $t$, stocks are sorted into tertiles based on realized jump returns during the holding period from July of year $t$ to June of year $t+1$. Stocks without jumps are assigned to a separate group. Then, within each of the four jump-sorted groups, stocks are further sorted into tertiles based on each of the four characteristics. The new tertile portfolios are computed by combining into a single equal-weighted portfolio the stocks with the same characteristic ranking across all four different jump groups. Table 8 reports the results. Once again, they are similar to those obtained before controlling for this variable. Size and liquidity, the two characteristics that have been fully realized in the form of jumps in previous results, continue to do so after controlling for jumps. They lack any significant predictive power after controlling for the jump effect.

To sum up findings thus far, the results obtained have shown that jumps have significant power to explain the premium on three of the four characteristics analyzed (size, btm and illiquidity). More importantly, in the case of size and illiquidity, which the preliminary analysis identified as the variables most strongly related to the presence of jumps (see Table 2), the continuous component prevents significant premiums from developing in total returns. The results obtained for the jump component in the Spanish 
market are therefore similar to those obtained for the US market, suggesting that the reaction to information captured by the jump component is more homogeneous across different markets than that captured by the continuous component, which may be more strongly tied to market-specific characteristics. While lying beyond the scope of this paper, cross-sectional and time-series analysis of the jump component in overall return patterns across markets might provide useful implications for portfolio management.

\section{Jumps, momentum and default risk}

The momentum results differ from those of the other characteristics by showing no significant premiums in the jump component while showing a significantly positive premium in the continuous component. Despite this widespread evidence, there is ongoing disagreement as to the source of momentum, with some scholars supporting risk-based explanations and others finding the most satisfactory explanation in behavioral finance theories.

The literature has linked the momentum effect to size (see Hong et al., 2000), due to the greater difficulty of small stocks and those commanding less attention from market analysts to fully incorporate information, and also to the btm ratio, (see Daniel and Titman, 1997) low btm ratios having been linked to asset pricing problems. Other characteristics with which the momentum effect has been related are the firm's industry (see Moskowitz and Grinblatt, 1999) and firm valuation problems (see Muga and Santamaría, 2007). More recently, Avramov et al. (2007) used US stock market data to show that momentum strategies will only achieve significant profits if strategies are constructed from low credit rating stocks, while Agarwal and Taffler (2008), using UK stock market data, concluded that the momentum effect is a direct consequence of market under-reaction to distress risk. However, Abinzano et al. (2014)), using a marketbased measure of default risk based on the Black-Scholes-Merton option-pricing model in four key European stock markets (France, Germany, Spain and the UK) find that default risk is not necessarily a characteristic of momentum strategies and cannot therefore explain momentum returns.

In this context, analysis of the components of total returns (JR and CR) can be very useful to test whether default risk is the source of the momentum effect, and thereby shed some light on this controversy. 
In order to compute the JR and CR components based on the default risk characteristic, in June of every year $t$, we sort the stocks using the BSM measure given by (10) and then compute the return for the holding period from July of year $t$ to June of year $t+1$. The stocks are then grouped into equal-weighted quintile portfolios. As a robustness check, we also compute LJR and LCR, and JR and CR controlling for jump risk and for realized jumps returns.

Tables 9 and 10 report the results. Although we find a default-risk premium of $6.54 \%$ per year ${ }^{16}$, it is largely attributable to the JR component, which accounts for $6.17 \%$, the contribution of the $\mathrm{CR}$ component being virtually null (0.37\%) and non-significant. These results differ completely from those reported for the momentum premium, where the JR component was negative and non-significant (-1.86\%) and the CR component positive and significant (10.47\%). Thus, the default risk and momentum premiums arise from different origins, the continuous return component in the case of momentum and the jump component in that of default risk ${ }^{17}$. Thus, momentum and default are unlikely to share the same source when one is consistent with explanations based on exposure to risk factors and the other with explanations based on investor behaviour. Thus, momentum and default risk are unlikely to share the same source when one is consistent with explanations based on exposure to risk factors and the other with explanations based on investor behaviour. These results appear to corroborate the conclusions reached by Abinzano et al. (2014) rather than those of Avramov et al. (2007) or Agarwal and Taffler (2008) and enable us to assert that default risk is not the source of momentum effect.

It is worth noting that, apart from momentum, the only characteristic of those analyzed in this paper that consistently displays a CR-related premium is btm, thereby corroborating the arguments put forward by Daniel and Titman (1997). Thus, insofar as btm is related with size, illiquidity and default risk, we can observe that momentum returns are also related to these three variables, but that the source of the returns is

\footnotetext{
16 This variable does not produce a significant quintile return spread because of the high variance of the quintile spread series. The jump component is nevertheless clearly significant.

${ }^{17}$ Despite the drawbacks of accounting measures mentioned in section 3, the robustness of the analysis was tested using Altman's Z Score. In line with other studies using accounting measures (see Agarwal and Taffler 2008), the default premium is negative, although, in our case, it lacks significance. However, as far as the main focus of the paper is concerned, since neither the jump component nor the continuous component is found to be significant, we are unable to conclude that the predictable component associated with default risk is realized in the same form (as price jumps or as continuous returns) as that associated with momentum. Thus, despite the change in the sign of the default premium, the main conclusion remains the same: default risk is unlikely to drive the momentum effect. These results are not shown but are available upon request from the authors.
} 
different. Nevertheless, when total returns are broken down into their components (JR and CR), only btm appears to share the same origin as the momentum effect. These results are highly revealing and suggest that the search for the origin of the momentum effect should be oriented towards btm-related variables, enabling us to cast aside numerous other explanations based on size, illiquidity or default risk.

In addition, the results obtained using the liquidity-adjusted measures (see Table 9) are similar to those provided by the unadjusted measures, notably in that the default risk premium is shown to be almost entirely due to the jump component. Furthermore, the analysis controlling for jump risk and for realized jump returns (see Table10) again reveals no significant positive premium attached to the CR component, in contrast to the findings for the momentum strategies. Thus, there is no difference between these and the results reported above.

\section{Conclusions}

The relationship between stock return predictability and various stock characteristics has been widely treated in the literature, which has offered risk-based as well as behavioural finance explanations. Jiang and Yao (2013) present a new way to unravel this controversy by separating the total stock return into its continuous and jump components. Their results for the US market indicate that stock return predictability is unlikely to be driven by a risk-based explanation.

In this paper, we analyze the Spanish stock market in order to test whether Jiang and Yao's (2013) findings are stock market-dependent. The Spanish stock market presents key differences in aspects such as the financial system, investor typology, stock characteristics and cultural dimensions. Our results show that these differences have major repercussions on the frequency and intensity of jumps, both of which are lower in the Spanish stock market. This finding is probably due to factors such as the larger average size of Spanish listed companies, their higher ownership concentration, and the stronger presence of institutional investors from the banking sector who have more stable relationships with firms. In spite of these differences, however, the main conclusions hold. Thus, our findings strengthen the robustness and generalizability of those of Jiang and Yao (2013), ours having been obtained in a setting that contrasts markedly with theirs in certain key market characteristics. Particularly, the jump component displays significant premiums in 3 of the 4 characteristics (size, btm and the 
illiquidity ratio), showing that the unexpected component of stock returns is related to lagged stock characteristics and is therefore difficult to reconcile with risk-based explanations.

In addition, analysis of the JR and CR components of total return has proven very useful for testing the default risk explanation for momentum, and thereby shedding some light on this controversy. Our results have shown that default risk premium is basically attributable to the jump component and the premium for the continuous component is virtually null. In the momentum premium, meanwhile, the role attributable to the jump component is non-significant, while that of the continuous component is positive and significant. This confirms the conclusions reached by Abinzano et al. (2014) and enables us to assert, in contradiction to the claims put forward by Avramov et al. (2007) and Agarwal and Taffler (2008), that default risk is not the source of momentum returns. In fact, the only other characteristic of those considered in this study which, like momentum, shows a significant premium attached to the continuous component is the btm ratio.

These very revealing findings suggest that we should direct the search for the source of momentum profits towards btm-related variables, in line with arguments presented by Daniel and Titman (1997), thereby ruling out numerous explanations based on size, illiquidity, or default risk. 


\section{Appendix A. The jump-day identification procedure.}

Following detection of a jump, we adopt the sequential approach used by Jiang and Yao (2013) to identify days with jumps. The specific sequence is as follows:

- Step 1: If the statistical jump test detects a jump during the testing period, we record the value of the jump statistic JT and continue to Step 2.

- Step 2: We replace each $\log$ return $r_{i}, i=1, \ldots, N$ in the testing period with the sample median, denoted by $r_{m}$ and perform the statistical jump test on each of the new $\log$ return series $\left\{r_{1}, \ldots, r_{i-1}, r_{m}, r_{i+1} \ldots, r_{N}\right\}$. In this way, a series of jump statistics $J T^{i}, i=1, \ldots, N$ are recorded. We use the median because it is robust to jumps. Furthermore, by replacing each return with the median instead of removing it from the sample, we are able to keep the sample size fixed and thereby maintain the power of the test.

- Step 3: We calculate the differences between the jump statistic in Step 1 and those in Step 2, thus obtaining a series of $J T-J T^{i},=1, \ldots, N$. The highest of all these values, $J T-J T^{j}$, identifies jump day $j$, and consequently the return on day $j, r_{j}$, is identified as the jump return. This is because replacement of return $r_{j}$ with the median causes the greatest reduction in the jump test statistic. This criterion is similar to the likelihood ratio test, since $r_{j}$ is the return in the testing period that contributes most to the rejection of the null hypothesis in the jump test in Step 1(i.e. the detection of jumps in the sample).

- Step 4: We substitute the jump return $r_{j}$ identified in the testing period with the median, thus obtaining a new sample of returns $\left\{r_{1}, \ldots, r_{j-1}, r_{m}, r_{j+1} \ldots, r_{N}\right\}$. We then repeat the process from Step 1 until no more jumps are detected by the JT statistic. Note that each time a jump day is detected, all the returns for identified jump days are set to the median over the sample period. 


\section{References}

Abinzano, I., Muga, L. and Santamaria, R. 2014. Is default risk the hidden factor in momentum returns? Some empirical results. Accounting and Finance, 54: 671698.

Abody, D., Lehavy, R. and Trueman B. 2010. Limited attention and the earnings announcement returns of past stock market winners. Review of Accounting Studies 15: 317-344.

Agarwal, V. and Taffler, R. 2008. Does financial distress risk drive the momentum anomaly? Financial Management 37: 461-484.

Aït-Sahalia, Y. and Jacod, J. 2009. Testing for jumps in a discretely observed process. The Annals of Statistics 37: 184-222.

Amihud, Y. 2002. Illiquidity and stock returns: cross-section and time-series effects. Journal of Financial Markets 5: 31-56.

Andersen, T.G., Benzoni, L. and Lund, J. 2002. An empirical investigation of continuoustime equity return models. Journal of Finance 57 (3): 1239-1284.

Andersen, T.G., Bollerslev, T. and Diebold, F.X. 2007. Roughing it up: including jump components in the measurement, modeling, and forecasting or return volatility. The Review of Economics and Statistics 89 (4):701-720.

Avramov, D., Chordia, T., Jostova, G. and Philipov, A. 2007. Momentum and credit rating. Journal of Finance 62 (5): 2503-2520.

Baker, M. and Wurgler, J. 2006. Investor Sentiment and the Cross-Section of Stock Returns. Journal of Finance 61(4): 1645-1680.

Barndorff-Nielsen, O.E. and Shephard, N. 2004. Power and bipower variation with stochastic volatility and jumps. Journal of Financial Econometrics 2:1-48.

Barndorff-Nielsen, O.E. and Shephard, N. 2006. Econometrics of testing for jumps in financial economics using bipower variation. Journal of Financial Econometrics 4: 1-30.

Bartov, E., Radhakrishnan, S. and Krinsky, I. 2000. Investor Sophistication and Patterns in Stock Returns After Earnings Announcements. The Accounting Review 75:43-63.

Bushee, B.J. 2001. Do Institutional Investors Prefer Near-Term Earnings over Long-Run Value?. Contemporary Accounting Research, 18(2):207-46.

Campbell, J., Lo, A. and MacKinlay, C. 1997. The econometrics of financial markets. Princeton, NJ: Princeton University Press.

Christensen, K., Oomen, R. and Podolskij, M. 2011. Fact or friction: jumps at ultra high frequency. Working paper, University of Aarhus.

Collins, D.W., Gong, G. and Hribar, P. 2003. Investor Sophistication and the Mispricing of Accruals. Review of Accounting Studies 8: 251-276.

Corredor P., Ferrer, E. and Santamaria R. 2013. Investor sentiment effect in stock markets: Stock characteristics or country-specific factors? International Review of Economics and Finance, 27:572-591.

Daniel K. and Titman, S. 1997. Evidence on the characteristics of cross sectional variation in stock returns. Journal of Finance 52: 1-33. 
Daniel, K., Hirshleifer, D. and Subrahmanyam, A. 1998. Investor Psychology and Security Market Under and Overreactions. Journal of Finance 53: 1839-1885.

Daniel, K., Hirshleifer, D. and Subrahmanyam, A. 2001. Overconfidence, Arbitrage, and Equilibrium Asset Pricing. Journal of Finance 56:921-965.

Fama E.F. and French K. 1992. The cross-section of expected stock returns. Journal of Finance 47:427-465.

Fama E.F. and French K. 1996. Multifactor explanations of asset pricing anomalies. Journal of Finance 51:55-84.

Griffin, J.M., Ji, X. and Martín, S. 2003. Momentum investing and business cycle risks: evidence from pole to pole. Journal of Finance, 58: 2515-2547.

Hirhsleifer, D.A. 2001. Investor Psychology and Asset Pricing, Journal of Finance 56: 1533-1597.

Hofstede, G. 2001. Culture's consequences: comparing values, behaviors, institutions, and organizations across nations. Sage Publication, Beverly Hills.

Hong, H., Lim, T. and Stein J.C. 2000. Bad news travel slowly: Size, analyst coverage, and the profitability of momentum strategies. Journal of Finance 55: 265-295.

Huang, X. and Tauchen, G. 2005. The relative contribution of jumps to total price variance. Journal of Financial Econometrics 3: 456-499.

Ince, O.S. and Porter, R.B. 2006. Individual equity return data from Thomson Datastream: Handle with care! Journal of Financial Research 29: 463-479.

Jagannathan, R. and Wang, Z. 1996. The Conditional CAPM and the Cross-Section of Expected Returns. Journal of Finance 51(1): 3-53.

Jegadeesh, N. and Titman S. 1993. Returns to buying winners and selling losers: Implications for stock market efficiency. Journal of Finance 48: 65-91.

Jiambalvo, J., Rajgopal, S. and Venkatachalam, M. 2002. Institutional ownership and the extent to which stock prices reflect future earnings. Contemporary Accounting Research 19(1): 117-45.

Jiang, G.J., and Oomen, R.C.A. (2008). Testing for jumps when asset prices are observed with noise - a "swap variance" approach. Journal of Econometrics 144: 352-370.

Jiang, G.J., and Yao, T. 2013. Stock price jumps and cross-sectional return predictability. Journal of Financial and Quantitative Analysis, 48: 1519-1544.

Jiang, G., Lee, Ch.M. and Zhang, Y. 2005. Information uncertainty and expected returns. Review of Accounting Studies 10: 185-221.

Lee, S. and Mykland, P. 2008. Jumps in financial markets: a new nonparametric test and jump dynamics. Review of Financial Studies 21: 2535-2563.

Merton, R.C. 1976. Option pricing when underlying stock returns are discontinuous. Journal of Financial Economics 3: 125-144.

Moskowitz T.J. and Grimblatt, M. 1999. Do industries explain momentum? The Journal of Finance, 54(4): 1249-1290.

Muga, L. and Santamaría, R. 2007. "New Economy" Firms and Momentum. Journal of Behavioral Finance 8: 109-120. 
Pan, J. 2002. The jump-risk premia implicit in options: evidence from an integrated time-series analysis of spot and option prices. Journal of Financial Economics 63: $3-50$.

Rouwenhorst, K.G. 1998. International momentum strategies. Journal of Finance 53: 267-284.

Schmeling, M. 2009. Investor sentiment and stock returns: some international evidence. Journal of Empirical Finance 16: 394-408.

Tauchen, G. and Zhou, H. 2011. Realized jumps on financial markets and predicting credit spreads Journal of Econometrics 160 (1): 102-118.

Vassalou, M. and Xing Y. 2004. Default risk in equity returns. Journal of Finance 49: 831-868.

Zhang, X. F. 2006. Information uncertainty and stock returns. Journal of Finance 61: 105-136. 
Table 1 Descriptive Statistics of stock price jumps

\begin{tabular}{|c|rrrrrr|r|}
\hline & $\mathbf{5 \%}$ & $\mathbf{2 5 \%}$ & mean & median & $\mathbf{7 5 \%}$ & $\mathbf{9 5 \%}$ & st. dev. \\
\hline J / year & 0.00 & 0.57 & 1.11 & 1.00 & 1.44 & 2.31 & 0.98 \\
(+) J /year & 0.00 & 0.41 & 0.84 & 0.75 & 1.09 & 1.77 & 0.81 \\
$(-)$ J / year & 0.00 & 0.05 & 0.26 & 0.19 & 0.37 & 0.80 & 0.30 \\
\hline J size \% & 4.55 & 6.90 & 9.87 & 8.82 & 11.64 & 19.32 & 4.65 \\
(+) J size \% & 4.55 & 6.88 & 9.52 & 8.62 & 11.60 & 18.62 & 4.44 \\
$(-)$ J size \% & -31.55 & -12.85 & -11.21 & -9.45 & -7.03 & -4.84 & 7.70 \\
\hline
\end{tabular}

This table reports the descriptive statistics (5th percentile, 1st quartile, mean, median, 3rd quartile, 95th percentile, and standard deviation) of jumps of individual stock prices. J / year ; (+) J /year ; (-) J / year is calculated in annual terms (from July of year $t$ to June of year $t+1$ ) as the ratio of total number of jumps / positive jumps/ negative jumps to the number of years the stock is in the sample. $\mathrm{J}$ size \% is the average in absolute value of all realized jumps. (+)J size \%/ (-) $\mathrm{J}$ size \% is the average of all positive /negative jumps. 
Table 2: Descriptive statistics of stock price jumps for portfolios sorted by stock characteristics

Size BTM Illiq Mom Size BTM Illiq Mom

\begin{tabular}{|c|c|c|c|c|c|c|c|c|}
\hline \multirow[b]{3}{*}{ Q1 } & \multirow{2}{*}{\multicolumn{4}{|c|}{$\mathrm{J} /$ year }} & \multirow{2}{*}{\multicolumn{4}{|c|}{$\mathrm{J}$ size \% }} \\
\hline & & & & & & & & \\
\hline & 1.24 & 1.06 & 0.76 & 1.17 & 0.12 & 0.10 & 0.08 & 0.11 \\
\hline Q2 & 1.32 & 1.18 & 1.12 & 1.07 & 0.10 & 0.08 & 0.09 & 0.08 \\
\hline Q3 & 1.12 & 1.18 & 1.28 & 1.11 & 0.09 & 0.09 & 0.11 & 0.09 \\
\hline Q4 & 1.03 & 1.05 & 1.43 & 1.12 & 0.08 & 0.09 & 0.10 & 0.09 \\
\hline Q5 & 0.92 & 1.07 & 1.28 & 1.12 & 0.07 & 0.11 & 0.12 & 0.11 \\
\hline Q5-Q1 & -0.32 & 0.02 & 0.52 & -0.05 & -0.05 & 0.01 & 0.04 & 0.00 \\
\hline \multirow[t]{2}{*}{$\mathrm{p}$-val } & 0.05 & 0.93 & 0.01 & 0.79 & 0.00 & 0.26 & 0.00 & 0.73 \\
\hline & \multicolumn{4}{|c|}{ (+) J /year } & \multicolumn{4}{|c|}{ (+) J size \% } \\
\hline Q1 & 1.04 & 0.77 & 0.50 & 0.94 & 0.12 & 0.09 & 0.07 & 0.11 \\
\hline Q2 & 1.03 & 0.94 & 0.88 & 0.85 & 0.10 & 0.08 & 0.08 & 0.08 \\
\hline Q3 & 0.92 & 0.91 & 0.98 & 0.82 & 0.09 & 0.08 & 0.10 & 0.08 \\
\hline Q4 & 0.77 & 0.81 & 1.18 & 0.90 & 0.07 & 0.09 & 0.11 & 0.08 \\
\hline Q5 & 0.68 & 0.93 & 1.05 & 0.87 & 0.07 & 0.11 & 0.12 & 0.10 \\
\hline Q5-Q1 & -0.37 & 0.15 & 0.54 & -0.07 & -0.05 & 0.02 & 0.05 & -0.01 \\
\hline \multirow[t]{2}{*}{$\mathrm{p}$-val } & 0.03 & 0.38 & 0.00 & 0.66 & 0.00 & 0.18 & 0.00 & 0.34 \\
\hline & \multicolumn{4}{|c|}{ (-) J/year } & \multicolumn{4}{|c|}{ (-) J size \% } \\
\hline Q1 & 0.21 & 0.28 & 0.25 & 0.23 & -0.16 & -0.11 & -0.09 & -0.10 \\
\hline Q2 & 0.27 & 0.24 & 0.25 & 0.22 & -0.09 & -0.09 & -0.12 & -0.10 \\
\hline Q3 & 0.32 & 0.27 & 0.30 & 0.29 & -0.09 & -0.09 & -0.12 & -0.10 \\
\hline Q4 & 0.24 & 0.23 & 0.25 & 0.22 & -0.12 & -0.08 & -0.10 & -0.10 \\
\hline Q5 & 0.28 & 0.15 & 0.24 & 0.25 & -0.08 & -0.12 & -0.11 & -0.11 \\
\hline Q5-Q1 & 0.06 & -0.14 & -0.02 & 0.03 & 0.08 & 0.00 & -0.01 & -0.01 \\
\hline p-val & 0.40 & 0.04 & 0.81 & 0.57 & 0.01 & 0.88 & 0.44 & 0.46 \\
\hline
\end{tabular}

This table reports the descriptive statistics (number of jumps in annual terms and the average jump size) for portfolios sorted on size, btm, illiquidity and momentum. P-val is the p-value associated with the two-sample t-tests for a difference in mean between the extreme quintiles. 
Table 3. Descriptive statistics for portfolios sorted on stock characteristics

\begin{tabular}{|c|cccc|}
\hline & SIZE & BTM & ILLIQUIDITY & MOMENTUM \\
& TR & TR & TR & TR \\
\hline Q1 & 0.1649 & 0.0758 & 0.0694 & 0.0885 \\
Q2 & 0.1207 & 0.0863 & 0.1362 & 0.0869 \\
Q3 & 0.1067 & 0.1101 & 0.0643 & 0.0713 \\
Q4 & 0.0882 & 0.1207 & 0.1495 & 0.0838 \\
Q5 & 0.0912 & 0.1976 & 0.1305 & 0.1746 \\
\hline Q5-Q1 & -0.0737 & 0.1218 & 0.0611 & 0.0861 \\
p-val & 0.2213 & 0.0131 & 0.2751 & 0.0246 \\
\hline
\end{tabular}

This table reports the average returns across stock characteristic quintiles. In June of each year t, stocks are sorted based on each stock characteristic to form equal-weighted portfolios. These portfolios are held from July t to June $t+1$. The table also shows the spread between the top and bottom quintiles and the p-value associated with the time series t-statistic for the return spreads.

Table 4. Jump returns and continuous returns across stock quintiles

\begin{tabular}{|c|cccccccc|}
\hline & \multicolumn{2}{|c}{ SIZE } & \multicolumn{2}{c}{ BTM } & \multicolumn{2}{c|}{ ILLIQUIDITY } & \multicolumn{2}{c|}{ MOMENTUM } \\
& JR & CR & JR & CR & JR & CR & JR & CR \\
\hline Q1 & 0.0931 & 0.0719 & 0.0391 & 0.0366 & 0.0118 & 0.0576 & 0.0793 & 0.0093 \\
Q2 & 0.0615 & 0.0592 & 0.0509 & 0.0355 & 0.0383 & 0.0979 & 0.0420 & 0.0449 \\
Q3 & 0.0648 & 0.0419 & 0.0447 & 0.0655 & 0.0637 & 0.0007 & 0.0434 & 0.0280 \\
Q4 & 0.0226 & 0.0657 & 0.0514 & 0.0694 & 0.0945 & 0.0550 & 0.0396 & 0.0442 \\
Q5 & 0.0264 & 0.0648 & 0.0777 & 0.1199 & 0.1110 & 0.0196 & 0.0606 & 0.1141 \\
\hline Q5-Q1 & -0.0667 & -0.0071 & 0.0386 & 0.0833 & 0.0992 & -0.0380 & -0.0187 & 0.1048 \\
p-val & 0.0006 & 0.8829 & 0.0079 & 0.0504 & 0.0011 & 0.4504 & 0.2563 & 0.0012 \\
\hline
\end{tabular}

This table reports the time series averages of jump returns (JR) and continuous returns (CR) for each characteristic-sorted quintile. Q5-Q1 is the spread between the top and bottom quintiles. P-val is the p-value associated with the time series t-statistic for the return spreads. 
Table 5. Jump returns and continuous returns across stock quintiles. Firms with jumps and without jumps

\begin{tabular}{|l|cccccc|}
\hline & \multicolumn{2}{|c}{ Total sample } & \multicolumn{2}{c|}{ Firms without jumps } & \multicolumn{2}{c|}{ Firms with jumps } \\
& return & p-value & return & p-value & return & p-value \\
\hline TR Size & -0.0737 & 0.2213 & -0.0423 & 0.4892 & -0.1246 & 0.0936 \\
TR btm & 0.1218 & 0.0131 & 0.1061 & 0.0557 & 0.1376 & 0.0150 \\
TR Illiquidity & 0.0611 & 0.2751 & -0.0149 & 0.7567 & 0.1325 & 0.1004 \\
TR Momentum & 0.0861 & 0.0246 & 0.0761 & 0.0828 & 0.0505 & 0.3766 \\
\hline
\end{tabular}

This table reports the average returns across stock characteristics quintiles. In June of each year t, stocks are sorted based on each stock characteristic to form equal-weighted portfolios. These portfolios are held from July $t$ to June $t+1$. The table shows the spread between the top and bottom quintiles and the $p$-value associated with the time series t-statistic for the total return spreads

Table 6. "liquidity-adjusted" Jump returns and "liquidity-adjusted" continuous returns across stock quintiles

\begin{tabular}{|c|cccccccc|}
\hline & \multicolumn{2}{|c}{ SIZE } & \multicolumn{2}{c}{ BTM } & \multicolumn{2}{c|}{ ILLIQUIDITY } & \multicolumn{2}{c|}{ MOMENTUM } \\
& LJR & LCR & LJR & LCR & LJR & LCR & LJR & LCR \\
\hline Q1 & 0.1069 & 0.0581 & 0.0558 & 0.0200 & 0.0178 & 0.0516 & 0.1101 & 0.0049 \\
Q2 & 0.0621 & 0.0586 & 0.0556 & 0.0307 & 0.0496 & 0.0866 & 0.0440 & 0.0423 \\
Q3 & 0.0765 & 0.0302 & 0.0534 & 0.0568 & 0.0688 & -0.0045 & 0.0500 & 0.0191 \\
Q4 & 0.0315 & 0.0567 & 0.0536 & 0.0672 & 0.1009 & 0.0486 & 0.0500 & 0.0348 \\
Q5 & 0.0349 & 0.0563 & 0.0946 & 0.1030 & 0.1403 & -0.0097 & 0.0669 & 0.1066 \\
\hline Q5-Q1 & -0.0720 & -0.0018 & 0.0389 & 0.0830 & 0.1225 & -0.0613 & -0.0209 & 0.1070 \\
p-val & 0.0048 & 0.9666 & 0.1248 & 0.0438 & 0.0070 & 0.2251 & 0.3610 & 0.0006 \\
\hline
\end{tabular}

This table reports the time series averages of "liquidity-adjusted" jump returns (LJR) and "liquidityadjusted" continuous returns (LCR) for each characteristic-sorted quintile. LJR is the originally identified jump return compounded with the stock returns over the subsequent four trading days to compensate for the potential reversion of stock prices, and LCR is the difference between total return and LJR. Q5-Q1 is the spread between the top and bottom quintiles. $\mathrm{P}$-val is the $\mathrm{p}$-value associated with the time series t-statistic for the return spreads. 
Table 7. Return spreads across stock quintiles: controlling for Jump risk

\begin{tabular}{|l|rl|rc|cc|rc|}
\hline & \multicolumn{2}{|c|}{ SIZE } & \multicolumn{2}{c|}{ BTM } & \multicolumn{2}{c|}{ ILLIQUIDITY } & \multicolumn{2}{c|}{ MOMENTUM } \\
\hline & return & p-value & return & p-value & return & p-value & return & p-value \\
\hline TR & -0.0066 & 0.8795 & 0.0498 & 0.1761 & 0.0293 & 0.4989 & 0.0389 & 0.1810 \\
\hline JR & -0.0700 & 0.0005 & 0.0343 & 0.0121 & 0.0869 & 0.0000 & -0.0105 & 0.3845 \\
CR & 0.0634 & 0.0876 & 0.0155 & 0.5844 & -0.0576 & 0.1265 & 0.0495 & 0.0715 \\
\hline LJR & -0.0757 & 0.0029 & 0.0359 & 0.0598 & 0.0998 & 0.0009 & -0.0095 & 0.5729 \\
LCR & 0.0691 & 0.0526 & 0.0139 & 0.6115 & -0.0705 & 0.0734 & 0.0485 & 0.0982 \\
\hline
\end{tabular}

Results controlled for jump risk using a double-sorting procedure. First, stocks are sorted into tertiles based on the jump risk measure JRisk. Then, within each tertile, stocks are further sorted into tertiles based on one of the four characteristics (size, btm, illiquidity ratio and momentum,). The new tertile portfolios are obtained by combining all the stocks with the same characteristic tertile ranking across three jump risk tertiles, and computing equal-weighted portfolios. This table shows the spread between the top and bottom quintiles and the p-value associated with the time series t-statistic for the return (TR, JR, CR, LJR and LCR) spreads.

Table 8. Return spreads across stock quintiles: controlling for Jump effects

\begin{tabular}{|l|rl|rl|rr|rr|}
\hline & \multicolumn{2}{|c|}{ SIZE } & \multicolumn{2}{c|}{ BTM } & \multicolumn{2}{c|}{ ILLIQUIDITY } & \multicolumn{2}{c|}{ MOMENTUM } \\
\hline & return & p-value & return & p-value & return & p-value & return & p-value \\
\hline TR & -0.0597 & 0.2595 & 0.1115 & 0.0109 & 0.0034 & 0.9372 & 0.0534 & 0.0536 \\
CR & 0.0131 & 0.7643 & 0.0795 & 0.0130 & -0.0685 & 0.1174 & 0.0553 & 0.0452 \\
\hline LCR & 0.0234 & 0.5517 & 0.0700 & 0.0179 & -0.0746 & 0.1067 & 0.0487 & 0.0683 \\
\hline
\end{tabular}

Results controlled by jump effect using a double-sorting procedure. In June of year $t$, stocks are sorted into tertiles based on realized jump returns during the holding period from July of year $t$ to June of year $t+1$. Stocks without jumps are assigned to a separate group. Then, within each of the four jump-sorted groups, stocks are further sorted into tertiles based on each of the four characteristics. The new tertile portfolios are computed by grouping into a single equal-weighted portfolio the stocks with the same characteristic ranking across the four different jump groups. This table shows the spread between the top and bottom quintiles and the p-value associated with the time series t-statistic for the return (TR, CR and LCR) spreads. 
Table 9. Jump returns and continuous returns across default risk quintiles

\begin{tabular}{|c|c|cc|cc|}
\hline & & & & & \\
& TR & JR & CR & LJR & LCR \\
\hline Q1 & 0.1313 & 0.0428 & 0.0886 & 0.0630 & 0.0684 \\
$\mathbf{Q 2}$ & 0.0891 & 0.0371 & 0.0520 & 0.0491 & 0.0400 \\
$\mathbf{Q 3}$ & 0.1186 & 0.0587 & 0.0599 & 0.0690 & 0.0496 \\
$\mathbf{Q 4}$ & 0.0908 & 0.0540 & 0.0369 & 0.0553 & 0.0356 \\
Q5 & 0.1968 & 0.1044 & 0.0923 & 0.1151 & 0.0817 \\
\hline Q5-Q1 & 0.0654 & 0.0616 & 0.0037 & 0.0521 & 0.0133 \\
p-val & 0.4606 & 0.0234 & 0.9655 & 0.0999 & 0.8816 \\
\hline
\end{tabular}

This table reports the time series averages of Total returns (TR), jump returns (JR), continuous returns (CR), "liquidity-adjusted" jump returns (LJR) and "liquidity-adjusted" continuous returns (LCR) for each quintile sorted on default risk. Q5-Q1 is the spread between the top and bottom quintiles. P-val is the pvalue associated with the time series t-statistic for the return spreads.

Table 10. Return spreads across default risk quintiles: controlling for Jump risk and jump effects

\begin{tabular}{|c|cc|cr|}
\hline & \multicolumn{2}{|c|}{ JUMP RISK } & \multicolumn{2}{c|}{ JUMP REALIZED } \\
\hline & return & p-value & return & p-value \\
\hline TR & 0.0291 & 0.6413 & 0.0392 & 0.5848 \\
\hline JR & 0.0422 & 0.0460 & - & - \\
CR & -0.0403 & 0.4903 & -0.0115 & 0.8628 \\
\hline LJR & 0.0455 & 0.0262 & - & - \\
LCR & -0.0163 & 0.7919 & -0.0081 & 0.8966 \\
\hline
\end{tabular}

Results controlled for jump risk and jump effects using a double-sorting procedure. First, stocks are sorted into tertiles based on the jump risk measure JRisk. Then, within each tertile, stocks are further sorted into tertiles based on default risk. The new tertile portfolios are obtained by combining all the stocks with the same characteristic tertile ranking across three jump risk tertiles, and computing equal-weighted portfolios. Then, in June of year $t$ stocks are sorted into tertiles based on realized jump returns during the holding period from July of year $t$ to June of year $t+1$. Stocks without jumps are assigned to a separate group. Then, within each of the four jump-sorted groups, stocks are further sorted into tertiles based on default risk. The new tertile portfolios are computed by grouping into a single equal-weighted portfolio the stocks with the same characteristic ranking across the four different jump groups. This table shows the spread between the top and bottom quintiles and the p-value associated with the time series t-statistic for the return (TR, JR, CR, LJR and LCR) spreads. 